\section{Could platelet-accumulating polyphenols prevent tumour metastasis?}

\section{Rita Negrão, Delfim Duarte, Raquel Costa and Raquel Soares}

We read with great interest the Review by Gay and Felding-Habermann (Contribution of platelets to tumour metastasis. Nature Rev. Cancer 11, 123-124 (2011)) ${ }^{1}$, which discussed the observation that cancer patients usually present signs of thrombosis, which is much more severe during metastatic progression. These authors suggest that platelets may protect tumours from immune elimination and enhance adhesion to blood vessels and tumour cell proliferation, which renders them useful for distant metastasis. According to these authors, platelets could be regarded as putative targets for cancer therapy. Platelets have a major role in haemostasis, by repairing minor vascular injuries and thrombosis $^{2}$. Platelets are also recognized by their important roles in the angiogenic process, given their release of pro-angiogenic factors such as vascular endothelial growth factor (VEGF), fibroblast growth factor 2 (FGF2; also known as bFGF), epidermal growth factor (EGF) and interleukin-1 $\beta$ (IL-1 $\beta$ ) upon activation.
We have been studying the effects on angiogenesis and inflammation of a group of naturally derived compounds, polyphenols, which have established anti-oxidant, antiinflammatory, anti-angiogenic and antitumour properties. The topical administration of two of these molecules, xanthohumol or iso xanthohumol (IXN), resulted in a reduction in systemic inflammatory factors and angiogenesis in granulation tissue in rat wound-healing assays ${ }^{3}$. Furthermore, we also demonstrated that xanthohumol reduces cancer progression and inflammation in a mouse xenograft breast cancer model ${ }^{4}$. Emerging evidence has revealed that these compounds can be internalized by platelets, in which they undergo further metabolism by platelet enzymatic activity $^{2}$. Interestingly, polyphenols may also interfere with platelet adhesion to the blood vessel wall, which is associated with the development of atherosclerotic plaques.

It is therefore conceivable that polyphenol accumulation within platelets in blood circulation may be considered as an anticancer mechanism of polyphenols. In agreement, the release of metabolized polyphenols by active platelets ${ }^{2}$ decreases local tumour cell viability, proliferation and invasion and increases apoptosis ${ }^{4}$. This effect may further be potentiated through the anti-inflammatory and anticancer properties that are characteristic of these compounds.

In conclusion, similar to anti-microbial pharmacological agents that are in clinical use, such as neutrophil-accumulating macrolides (which are then released in an active form at the infection site ${ }^{5}$ ), the accumulation of polyphenols in platelets could prevent tumour metastasis.

Rita Negrão, Delfim Duarte, Raquel Costa and Raquel Soares are at the Department of Biochemistry (U38), Faculty of Medicine, University of Porto, Al. Prof. Hernâni Monteiro, 4200-319 Porto, Portugal. Correspondence to R.S. e-mail: raqsoa@med.up.pt doi: 10.1038/nrc3004-c1

1. Gay, L. J. \& Felding-Habermann, B. Contribution of platelets to tumour metastasis. Nature Rev. Cancer 11, 123-134 (2011).

2. Wright, B., Gibson, T., Spencer, J., Lovegrove, J. A. \& Gibbins, J. M. Platelet-mediated metabolism of the dietary flavonoid, Quercetin. PLOS ONE 5, e9673 (2010).

3. Negrão, R. et al. Angiogenesis and inflammation signaling are targets of beer polyphenols on vascular cells. J. Cell Biochem. 111, 1270-1279 (2010).

4. Monteiro, R. et al. Xanthohumol inhibits inflammatory factor production and angiogenesis in breast cancer xenografts. J. Cell Biochem. 104, 1699-1707 (2008).

5. Mandell, G. L. \& Coleman, E. Uptake, transport, and delivery of antimicrobial agents by human polymorphonuclear neutrophils. Antimicrob. Agents Chemother. 45, 1794-1798 (2001).

Competing interests statement

The authors declare no competing financial interests. 